\title{
Differential Effects of Ursolic Acid and Oleanolic Acid on Mitochondrial ATP Generation in H9c2 Cardiomyocytes and Lipopolysaccharide-Induced Cell Proliferation in Mouse Splenocytes: Yang versus Yin
}

\author{
Hoi Yan Leung, Kai Chit Cheung, Kam Ming Ko* \\ Division of Life Science, Hong Kong University of Science \& Technology, Hong Kong, China \\ Email: ^bcrko@ust.hk
}

How to cite this paper: Leung, H.Y., Cheung, K.C. and Ko, K.M. (2021) Differential Effects of Ursolic Acid and Oleanolic Acid on Mitochondrial ATP Generation in H9c2 Cardiomyocytes and Lipopolysaccharide-Induced Cell Proliferation in Mouse Splenocytes: Yang versus Yin. Chinese Medicine, 12, 37-46. https://doi.org/10.4236/cm.2021.123005

Received: July 2, 2021

Accepted: August 31, 2021

Published: September 3, 2021

Copyright ( 2021 by author(s) and Scientific Research Publishing Inc. This work is licensed under the Creative Commons Attribution International License (CC BY 4.0).

http://creativecommons.org/licenses/by/4.0/

\begin{abstract}
In the present study, two cell-based systems for assessing Yang and Yin activities were for the first time used to investigate the effect of ursolic acid (UA) and oleanolic acid (OA). The results indicated that while UA was only active in the Yang assay, OA produced activity in the Yin assay. The Yang/Yin activity of UA/OA may be attributed to their distinct molecular structures, which confer their differential ability to interact with mitochondrial membrane or cellular membrane lipids, with resultant membrane fluidization and potentiation of biological responses.
\end{abstract}

\section{Keywords}

Yang, Yin, Ursolic Acid, Oleanolic Acid, Mitochondrial ATP Generation, Splenocytes

\section{Introduction}

According to traditional Chinese medicine theory, diseases are caused by an imbalance of Yang and Yin in the body, and Chinese tonifying herbs have long been used to correct the bodily imbalance of Yang and Yin by Yang-invigoration and Yin-nourishment, respectively [1]. Ursolic acid (UA) and oleanolic acid (OA), which are active components of Cynomorri herba (a Yang-invigorating herb) and Ligustri fructus (a Yin-nourishing herb), respectively [2], are triterpenoids also found in edible plants and other herbs [3]. Although UA and OA 
are stereoisomers (see Figure 1), they have been shown to share some common pharmacological properties [3]. Recent studies in our laboratory have developed cell-based pharmacological assays for Yang-invigorating and Yin-nourishing activities in Chinese tonifying herbs [4] [5]. In the present study, we aimed for the first time to investigate whether the difference in structural features between UA and $\mathrm{OA}$ is causally related to the elicitation of Yang and Yin activity, as assessed by mitochondrial ATP generation capacity in cultured H9c2 cells and lipopolysaccharide (LPS)-induced proliferation in isolated mouse splenocytes, respectively. The possible involvement of alterations in cellular/mitochondrial membrane fluidity in causing Yang and Yin activity was examined by pre-incubating cells with cholesterol which reduces membrane fluidity [6].

\section{Materials and Methods}

\subsection{Reagents}

Fetal bovine serum (FBS) and Dulbecco's modified Eagle's medium (DMEM) were obtained from Life Technologies (Grand Island, NY, USA). RPMI 1640 medium, L-malic acid, adenosine 5' diphosphate sodium salt (ADP), adenosine 5' triphosphate disodium salt (ATP), dimethyl sulfoxide (DMSO), 3-[4,5-dimethylthiazol-2-yl]-2,5-diphenyl tetrazolium bromide (MTT), digitonin, cholesterol, and LPS, were purchased from Sigma-Aldrich Co (St. Louis, MO, USA). UA and OA were procured from Bidepharm (Shanghai, China) and Shanghai Macklin Biochemical Co., Ltd. (Shanghai, China), respectively. Sodium pyruvate and bovine serum albumin (BSA) were obtained from Santa Cruz Biotechnology Inc. (Santa Cruz, CA, USA). ATPlite luminescence assay kit was bought from PerkinElmer (Waltham, MA, USA). Bio-Rad protein assay dye reagent concentrate was obtained from Bio-Rad (Hercules, CA, USA). All other chemicals were of analytical grade.

\subsection{Cell Culture}

A H9c2 cell line derived from embryonic rat heart tissue was purchased from American Type Culture Collection (Rockville, MD, USA). H9c2 cells were cultured in DMEM supplemented with $10 \%(\mathrm{v} / \mathrm{v})$ heat-inactivated FBS (HIFBS) and $1 \mathrm{mM}$ sodium pyruvate. Cells were cultured in an atmosphere of $5 \% \mathrm{CO}_{2}$ at $37^{\circ} \mathrm{C}$ in a $100 \mathrm{~mm}$ culture plate. The medium was replaced every $2-3$ days and cells were split at a subcultivation ratio of 1:10 2 - 3 days after medium replacement.

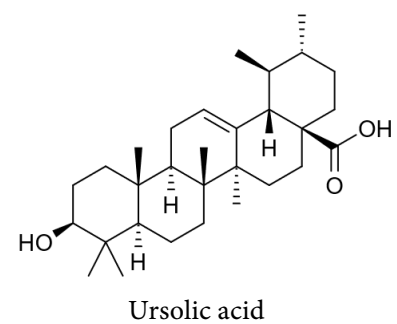

Ursolic acid

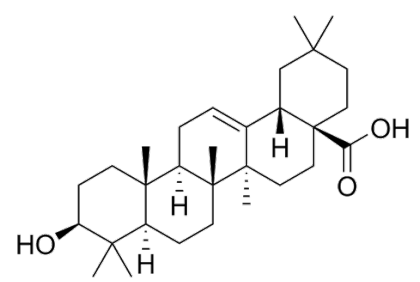

Oleanolic acid

Figure 1. Chemical structures of ursolic acid and oleanolic acid. 


\subsection{ATP Generation Capacity (ATP-GC) Assay}

Drug pre-incubation and ATP Generation

H9c2 cells were seeded in four 24 -well plates $(25,000$ cells $/ 0.5 \mathrm{~mL})$. After stable attachment, the cells were pre-incubated for 4 hours with a medium containing $2-10 \mu \mathrm{M}$ of UA or OA [dissolved in DMSO, $0.2 \%(\mathrm{v} / \mathrm{v})$ final concentration]. Following the incubation, the cells were washed with phosphate-buffered saline (PBS, $200 \mu \mathrm{L} /$ well; $136 \mathrm{mM} \mathrm{NaCl}, 2.7 \mathrm{mM} \mathrm{KCl}, 8.1 \mathrm{mM} \mathrm{Na} \mathrm{HPO}_{4}, 1.5 \mathrm{mM}$ $\mathrm{KH}_{2} \mathrm{PO}_{4}, \mathrm{pH} 7.2$ ) and treated with $50 \mu \mathrm{g} / \mathrm{mL}$ digitonin at $37^{\circ} \mathrm{C}$ for 3 minutes, except for the protein measurement plate, which was stored at $4^{\circ} \mathrm{C}$ until protein determination. For the plate used for measuring basal ATP level, cells were incubated at $4^{\circ} \mathrm{C}$ with ATP incubation buffer $\left(120 \mathrm{mM} \mathrm{KCl,} 5 \mathrm{mM} \mathrm{KH}_{2} \mathrm{PO}_{4}, 2 \mathrm{mM}\right.$ EGTA, $10 \mathrm{mM}$ HEPES, $0.1 \mathrm{mM} \mathrm{MgCl}_{2}, 0.5 \%$ BSA, pH7.4, $150 \mu \mathrm{L} /$ well) and 30\% $(\mathrm{w} / \mathrm{v})$ perchloric acid (PCA, $30 \mu \mathrm{L} /$ well). For the plates used for ATP generation, cells were supplemented with $0.18 \mathrm{mM}$ ADP, $5 \mathrm{mM}$ pyruvate, and $15 \mathrm{mM}$ L-malic acid (all dissolved in ATP incubation buffer, $100 \mu \mathrm{L}$ for each reagent/well) and incubated at $37^{\circ} \mathrm{C}$ for 7.5 and 15 minutes, respectively, followed by the addition of $30 \%$ PCA $(60 \mu \mathrm{L} /$ well). The plates (for measuring basal ATP and ATP generation) were then centrifuged at $540 \times \mathrm{g}$ for 20 minutes at $4^{\circ} \mathrm{C}$. After centrifugation, aliquots $(80 \mu \mathrm{L})$ of supernatants were neutralized with $60 \mu \mathrm{L}$ $1.4 \mathrm{M} \mathrm{KHCO}_{3}$ in $1.5 \mathrm{~mL}$ micro-centrifuge tubes, followed by vortex mixing and centrifugation at $2150 \times \mathrm{g}$ for $10 \mathrm{~min}$.

ATP assay

To account for ATP contamination in the ADP preparation, an ADP blank was prepared by aliquoting ADP, pyruvate, L-malic acid and 30\% PCA, as described earlier, to $1.5 \mathrm{~mL}$ in a micro-centrifuge tube, followed by vortex mixing and centrifugation at $20,000 \times \mathrm{g}$ for $5 \mathrm{~min}$. ATP standards (ranging from 0.0005 $1.0 \mathrm{mM})$ were prepared by 10 -fold serial dilutions. Aliquots $(300 \mu \mathrm{L})$ of standard solutions were added to $60 \mu \mathrm{L} 30 \%$ PCA, followed by vortex mixing, and $80 \mu \mathrm{L}$ of the standard solutions, as well as the ADP blank, were neutralized with $60 \mu \mathrm{L}$ 1.4 $\mathrm{M} \mathrm{KHCO}_{3}$, followed by vortex mixing and centrifugation at $20,000 \times \mathrm{g}$ for 5 min. Fifty micro-liters of ATP standards $(0.0005-1 \mathrm{mM})$ or tested samples were applied to 96 -well white plates with $50 \mu \mathrm{L} 10$-fold diluted luciferase enzyme prepared in luciferase dilution buffer (250 mM Tricine, $5 \mathrm{mM}$ EDTA (free acid), $5 \mathrm{mM}$ dithiothreitol, $50 \mathrm{mM} \mathrm{MgSO}$, pH 7.8 adjusted by $1 \mathrm{M} \mathrm{KOH}$ ). The luminescence was read using a microplate reader. ATP content was estimated from an ATP standard calibration curve. ATP-GC was expressed in nmol/mg protein and the area under the curve (AUC1) of the graph plotting ATP generation against incubation time was then computed (Figure 2). These AUC1 values were used to calculate the percentage of control (\% Control1), and then the AUC2 of the graph plotting \% Controll against incubation time was computed. These AUC2 values were used to calculate the percentage of control (\% Control2) for comparison.

Protein Measurement 


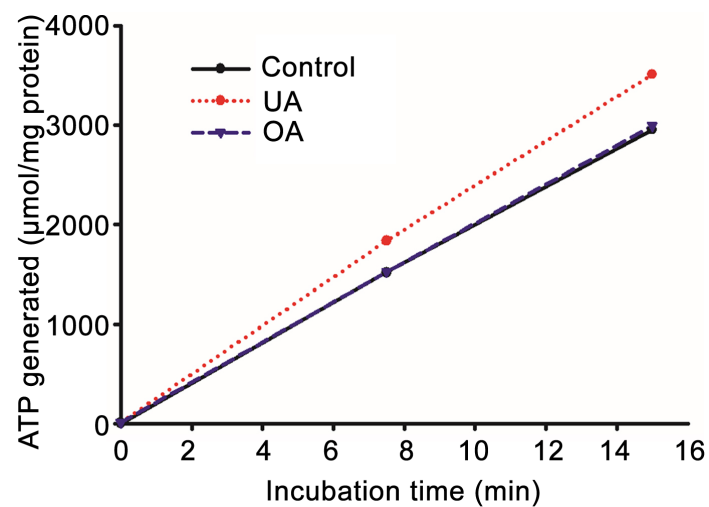

Figure 2. Time course of ATP generation in the presence or absence of UA/OA.

Proteins were extracted from the cultured cells using $0.1 \%$ Triton X in PBS ( $200 \mu \mathrm{L} /$ well), followed by 10 -minutes of shaking at room temperature. Aliquots $(10 \mu \mathrm{L})$ of samples, as well as BSA standards, were mixed with working protein-dye solution $(200 \mu \mathrm{L} /$ well $)$ and the reaction mixtures were allowed to stand at room temperature for 5 minutes. The absorbance of the samples was then read at $600 \mathrm{~nm}$.

\subsection{LPS-Induced Splenocyte Proliferation}

Splenocyte isolation

Splenic tissue obtained from adult female ICR mice (25 - $40 \mathrm{~g}$ body weight) was pressed through a mesh stainless steel sieve using a glass pestle in $25 \mathrm{~mL}$ RPMI-1640 medium to obtain a single-cell suspension. The cells were placed on ice for $5 \mathrm{~min}$, and the suspension was centrifuged at $600 \times \mathrm{g}$ for $5 \mathrm{~min}$, followed by washing with red blood cell lysis buffer $(0.1 \mathrm{M}$ Tris- $\mathrm{HCl}, \mathrm{pH} 7.2,0.8 \%$ $\mathrm{NH}_{4} \mathrm{Cl}$ ) and RPMI-1640 medium. After centrifugation, the pellet was resuspended in RPMI-1640 medium supplemented with 10\% HIFBS for cell counting, using $0.4 \%$ trypan blue. The cells were then adjusted to $5 \times 10^{6}$ cells $/ \mathrm{mL}$ and 80 $\mu \mathrm{L}$ of cell suspensions were seeded in each well of a 96-well plate.

Drug incubation and LPS stimulation in vitro

UA or OA (dissolved in DMSO) was added at final concentrations of 3 - 100 $\mu \mathrm{M}$. LPS was added at final concentrations of $0.5,1,2,4$, and $7.5 \mu \mathrm{g} / \mathrm{mL}$. Splenocytes were then cultured for $72 \mathrm{~h}$ at $37^{\circ} \mathrm{C}$ in a humidified atmosphere of $5 \%$ $\mathrm{CO}_{2}$ in air. Thereafter, the extent of cell proliferation was assessed.

MTT-based cell proliferation assay

An aliquot $(10 \mu \mathrm{L})$ of MTT $(5 \mathrm{mg} / \mathrm{mL}$ in PBS) was added to each well. After 4 $\mathrm{h}$ of incubation, $100 \mu \mathrm{L}$ of solubilization buffer (10\% sodium dodecyl sulfate, $45 \%$ dimethylformamide, $\mathrm{pH} 4.7$ adjusted by glacial acetic acid) was added, and the mixtures were incubated in $5 \% \mathrm{CO}_{2}$ at $37^{\circ} \mathrm{C}$ overnight to dissolve the colored crystals. The extent of splenocyte proliferation was determined by measuring the absorbance at $600 \mathrm{~nm}$ using a microplate reader. The extent of LPS-stimulated proliferation of isolated splenocytes was estimated by computing the AUC of a graph plotting the percentage of initial absorbance (mean absorbance of cells 
stimulated with LPS/mean absorbance of cells not stimulated with LPS $\times 100 \%$ ) against LPS concentration (Figure 3). The extent of LPS-stimulated proliferation of isolated splenocytes was estimated by comparison with the control.

\subsection{Cholesterol Pre-Incubation}

H9c2 cells

Cultured cells were pre-incubated with cholesterol (dissolved in absolute ethanol) at a final concentration of $10 \mu \mathrm{M}$ for $4 \mathrm{~h}$. Cells were then washed with PBS buffer and subjected to drug pre-incubation, which was followed by the measurement of ATP generation.

Mouse splenocytes

The cell suspension was pre-incubated with absolute ethanol or $10 \mu \mathrm{M}$ cholesterol (final concentration, dissolved in absolute ethanol) in RPMI-1640 medium supplemented with 10\% HIFBS for 4 hours, followed by washing with RPMI-1640 medium supplemented with 10\% HIFBS and then centrifuging at $540 \times \mathrm{g}$ for 7 minutes. The cells were then adjusted to $5 \times 10^{6}$ cells $/ \mathrm{mL}$ and were seeded in a $96-$ well plate.

\subsection{Statistical Analysis}

Data, which were expressed as mean \pm SEM, were analyzed by one-way ANOVA, followed by Tukey's test to detect significant differences between groups when $\mathrm{p}<0.05$.

\section{Results and Discussion}

In the present study, two cell-based assays for assessing Yang-invigorating and Yin-nourishing activities in Chinese tonifying herbs were used to investigate the effects of UA and OA, which are stereoisomers and biologically active triterpenoids found in Cynormorii herba (a Yang-invigorating herb) and Ligustri fructus (a Yin-nourishing herb) in traditional Chinese medicine. The cytotoxicity of UA/OA was tested, and no detectable toxicity was detected up to a concentration of $10 \mu \mathrm{M}$ for 4 hours incubation on H9c2 cell line (data not

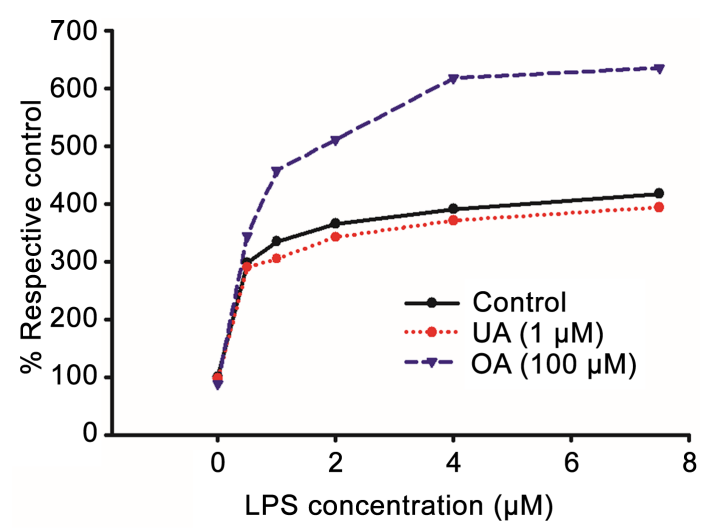

Figure 3. Concentration-dependent of LPS-induced splenocyte proliferation in the presence or absence of UA/OA. 
shown). For 72-hour incubation on splenocyte, there was no detectable toxicity was detected up to a concentration of 1 and $100 \mu \mathrm{M}$ of UA and OA, respectively (data not shown). Whereas UA incubation caused a concentration-dependent increase (up to 24\%) in ATP-GC in H9c2 cells, incubation with OA produced no detectable effect (Figure 4). The stimulation of ATP-GC by UA was completely abrogated by cholesterol pre-incubation (Figure 5). On the other hand, UA incubation did not produce any detectable effect on LPS-stimulated splenocyte proliferation, but incubation with OA caused a concentration-dependent increase in the extent of splenocyte proliferation (13\% - 36\%) upon LPS stimulation (Figure 6). The stimulatory effect of OA was completely suppressed by cholesterol pre-incubation (Figure 7). The differential effects of UA and OA on ATP-GC stimulation (Yang) and immunomodulation (Yin) are consistent with the characteristic pharmacological actions of their herbs of origin, namely, $C y$ nomorii herba (Yang) and Ligustri fructus (Yin). However, the molecular basis underlying the differential effect of UA and OA on the Yang and Yin cell-based assays remains elusive.

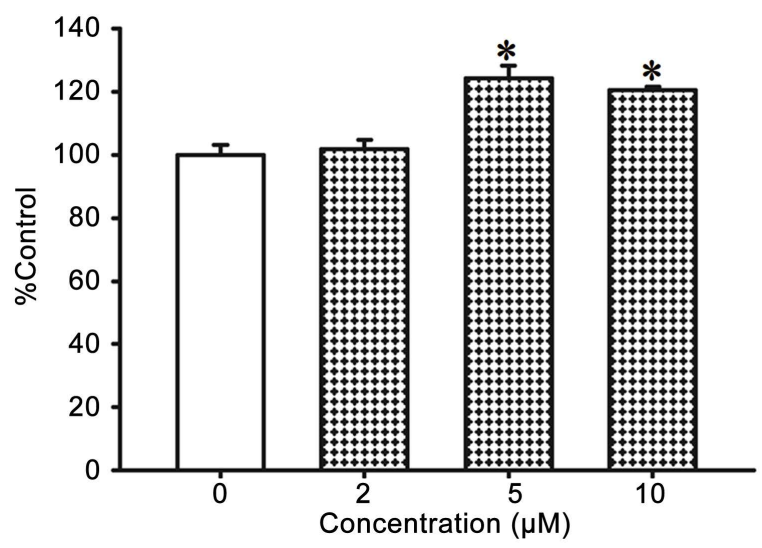

(a)

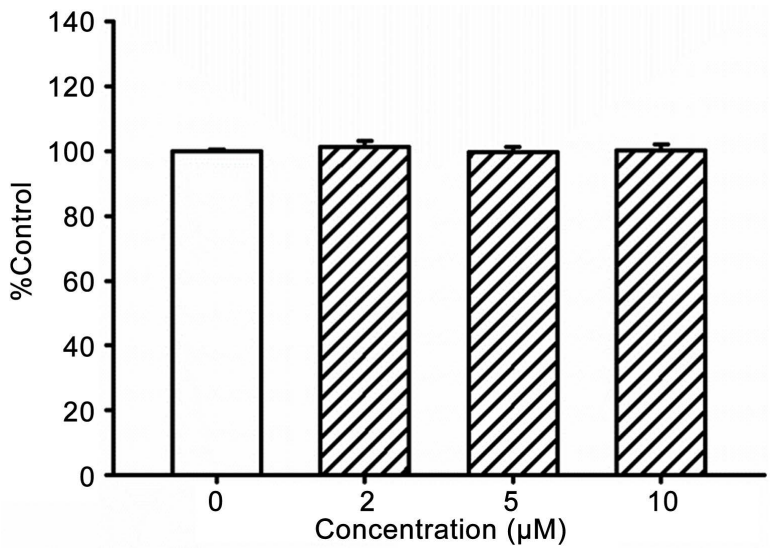

(b)

Figure 4. The effect of UA or OA on ATP-GC in H9c2 cells. ATP-GC was measured as described in Materials and Methods. Values given are the percent control, when compared with the sample without drug incubation. The control value (AUC2) is $750.00 \pm$ 3.50. ${ }^{*}$ Significantly different from the control. (a) UA; (b) OA. 


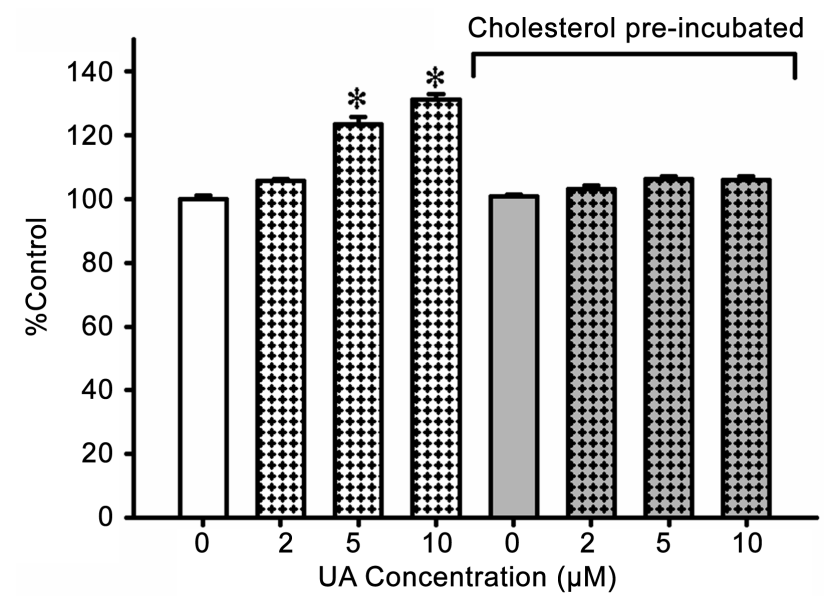

Figure 5. The effect of cholesterol pre-incubation on ATP-GC in UA-incubated H9c2 cells. Cholesterol pre-incubation was performed as described in Materials and Methods. ${ }^{\star}$ Significantly different from the control.

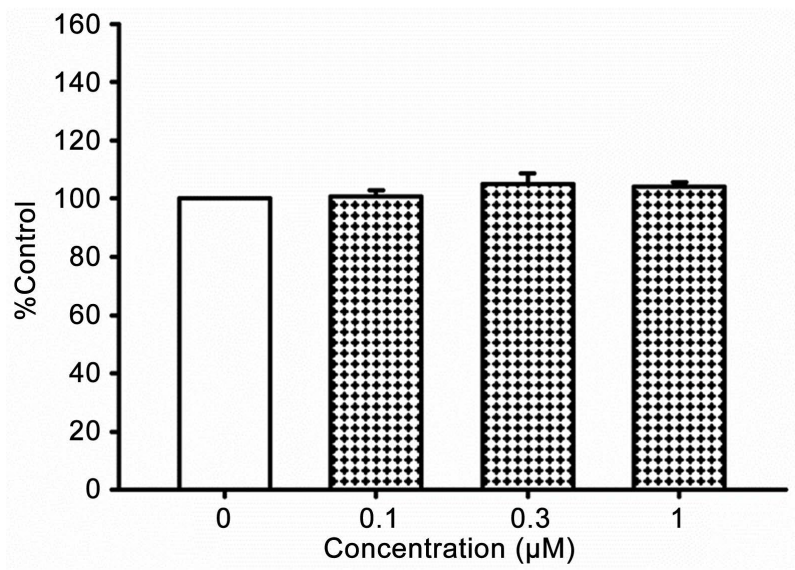

(a)

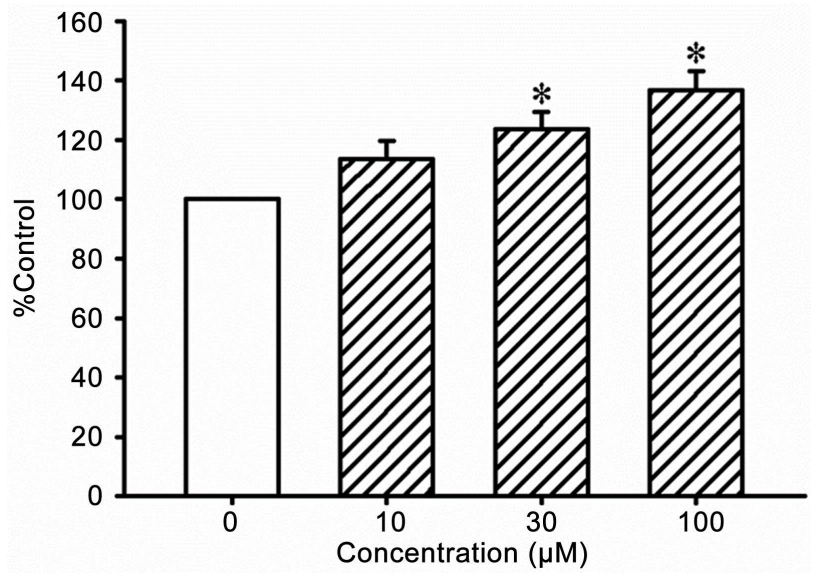

(b)

Figure 6. The effect of UA or OA on cell proliferation in LPS-stimulated mouse splenocytes. The LPS-induced proliferation of mouse splenocytes was measured as described in Materials and Methods. Values given are the percent control, when compared with the sample without drug incubation. The control value (AUC) is $2779.90 \pm 37.67$. ${ }^{\star}$ Significantly different from the control. (a) UA; (b) OA. 


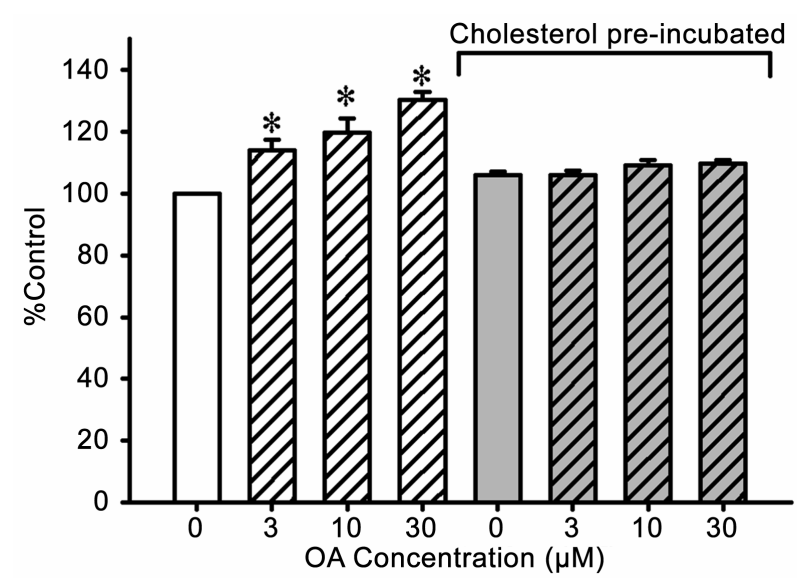

Figure 7. The effect of cholesterol pre-incubation on cell proliferation in OA-incubated and LPS-stimulated mouse splenocytes. Cholesterol pre-incubation was performed as described in Materials and Methods. ${ }^{*}$ Significantly different from the control.

The increase in ATP generation caused by UA, but not OA, may be due to the minor structural difference of the methyl group positioning on the ring $\mathrm{E}$ of both compounds (see Figure 1). As ATP generation occurs mainly at the inner membrane of mitochondria, UA may increase membrane fluidity and thus mitochondrial electron transport, with a resultant increase ATP generation, as was shown to be the case with $\beta$-sitosterol [7]. When incorporated into the mitochondrial inner membrane, the positioning of the methyl group in UA may occupy less space than its counterpart in OA. As a result, only UA can cause the fluidization of membrane lipids in the mitochondria. This postulation is supported by the observation that the stimulatory effect of UA on ATP-GC was completely abrogated by cholesterol pre-incubation, which presumably prevented the membrane fluidization.

OA, but not UA, produced a stimulatory effect on LPS-stimulated splenocyte proliferation. This effect was suppressed by pre-incubation with cholesterol. Conceivably, UA and OA can specifically influence the fluidity of mitochondrial inner membrane and plasma membranes, respectively, which in turn could alter the activity of transmembrane proteins such as electron transport components and the TLR4/MD-2 receptor complex. The relationship between membrane fluidity and receptor activity has been demonstrated by the observation that a shift in equilibrium towards the active form of the receptor can be caused by an increase in membrane fluidity [8]. As the inner membrane of mitochondria has a similar phospholipid composition to bacterial membranes as described in the endosymbiotic theory [9], UA may fluidize the mitochondrial inner membrane by its structural character, which differs from that of OA. In contrast, OA can fluidize the plasma membrane.

OA might have a direct interaction with the LPS-binding receptor or the signal transduction pathway that stimulates cell proliferation. In this regard, the small molecule euodenine A has been shown to act as an agonist of the human TLR4 receptor [10]. A study using molecular modeling predicts that UA may 
have the ability to bind to the TLR4-MD2 complex and alter its conformation, thereby decreasing the receptor's signal transduction activity [11]. Although an inhibitory effect of UA was not observed in the present study, OA may directly interact with the TLR4-MD2 complex and enhance signal transduction.

\section{Conclusion}

In conclusion, the Yang and Yin activity of UA and OA, as assessed by cell-based assays, may be attributed to their distinct molecular structures, which determine their ability to interact with mitochondrial membrane or cellular membrane lipids, with resultant membrane fluidization and potentiation of biological responses.

\section{Conflicts of Interest}

The authors declare no conflicts of interest regarding the publication of this paper.

\section{References}

[1] Leong, P.K., Chen, J. and Ko, K.M. (2018) Development of Chinese Herbal Health Products for the Prevention of Aging-Associated Diseases. In: Mandal, S.C., Mandal, V. and Konishi, T., Eds., Natural Products and Drug Discovery: An Integrated Approach, Elsevier, Amsterdam, 73-104. https://doi.org/10.1016/B978-0-08-102081-4.00004-6

[2] Yim, T.K. and Ko, K.M. (2002) Antioxidant and Immunomodulatory Activities of Chinese Tonifying Herbs. Pharmaceutical Biology, 40, 329-335.

https://doi.org/10.1076/phbi.40.5.329.8457

[3] Liu, J. (1995) Pharmacology of Oleanolic Acid and Ursolic Acid. Journal of Ethnopharmacology, 49, 57-68. https://doi.org/10.1016/0378-8741(95)90032-2

[4] Leong, P.K. and Ko, K.M. (2019) Biomarkers for Quality Control of Chinese Tonifying Herbs and Herbal Health Products. In: Ghosh, D. and Mukherjee, P.K., Eds., Natural Medicines: Clinical Efficacy, Safety, and Quality, CRC Press (Taylor \& Francis Group), Boca Raton, Chapter 7, 127-138.

https://doi.org/10.1201/9781315187853-7

[5] Wong, H.S., Leung, H.Y. and Ko, K.M. (2011) "Yang-Invigorating" Chinese Tonic Herbs Enhance Mitochondrial ATP Generation in H9c2 Cardiomyocytes. Chinese Medicine, 2, 1-5.

[6] Cooper, R.A. (1978) Influence of Increased Membrane Cholesterol on Membrane Fluidity and Cell Function in Human Red Blood Cells. Journal of Supramolecular Structure, 8, 413-430. https://doi.org/10.1002/jss.400080404

[7] Wong, H.S., Leong, P.K., Chen, J., Leung, H.Y., Chan, W.M. and Ko, K.M. (2016) $\beta$-Sitosterol Increases Mitochondrial Electron Transport by Fluidizing Mitochondrial Membranes and Enhances Mitochondrial Responsiveness to Increasing Energy Demand by the Induction of Uncoupling in C2C12 Myotubes. Journal of Functional Foods, 23, 253-260. https://doi.org/10.1016/j.jff.2016.02.045

[8] Yoshida, K., Nagatoishi, S., Kuroda, D., Suzuki, N., Murata, T. and Tsumoto, K. (2019) Phospholipid Membrane Fluidity Alters Ligand Binding Activity of a G Protein-Coupled Receptor by Shifting the Conformational Equilibrium. Biochemistry, 58, 504-508. https://doi.org/10.1021/acs.biochem.8b01194 
[9] Gould, S.B. (2018) Membranes and Evolution. Current Biology, 28, R367-R420. https://doi.org/10.1016/j.cub.2018.01.086

[10] Neve, J., Wijesekera, H., Duffy, S., Jenkins, I., Ripper, J., Teague, S., et al. (2014) Euodenine A: A Small-Molecule Agonist of Human TLR4. Journal of Medicinal Chemistry, 57, 1252-1275. https://doi.org/10.1021/jm401321v

[11] Niu, X., Yu, Y., Guo, H., Yang, Y., Wang, G., Sun, L., Gao, Y., Yu, Z. and Wang, H. (2018) Molecular Modeling Reveals the Inhibition Mechanism and Binding Mode of Ursolic Acid to TLR4-MD2. Computational and Theoretical Chemistry, 1123, 73-78. https://doi.org/10.1016/j.comptc.2017.11.016 